\title{
IoTA: IoT Automated SIP-based Emergency Call Triggering System for general eHealth purposes.
}

\author{
Foteini Andriopoulou,Theofanis Orphanoudakis \\ School of Science and Technology \\ Hellenic Open University \\ Patras, 26335, Greece \\ Email:fandriopoulou@eap.gr,fanis@eap.gr
}

\author{
Tasos Dagiuklas \\ School of Engineering, Computer Science and Informatics \\ London South Bank University \\ 103 Borough Road,London SE1 OAA \\ Email:tdagiuklas@lsbu.ac.uk
}

\begin{abstract}
The expansion of Internet of Things (IoT) and the evolution in communication technologies have enabled homes, cars even whole cities to be network connected. However, during an emergency incident, IoT devices have not been used to trigger emergency calls directly to healthcare providers mainly due to their constrained capabilities and lack of support session-oriented communications. Moreover, emergency services are currently offered by public safety stakeholders that do not support call triggering by IoT devices. This paper proposes IoTA framework which enables IoT devices to generate automatically emergency calls and support bi-directional communication sessions between healthcare providers and end users. The IoTA framework incorporates intelligent algorithms for processing and evaluating emergency events from various devices and performs emergency calls immediately after the occurrence of an event. The healthcare providers can interact with the IoTA framework requesting continuous real-time sensor data. A prototype implementation and initial evaluation results are presented as a proof of concept for people suffering from diverse chronic diseases. Experimental results have shown that the proposed framework can be considered as a promising solution for detecting, reporting emergency events, eliminating the hoax calls and responding swiftly saving lives.
\end{abstract}

Keywords-emergency calls; Internet of Things; IoT; sensor devices; IP communication session; SIP based calls; eHealth; seizure

\section{INTRODUCTION}

As every device and appliance can potentially be connected to the Internet, Internet of Things (IoT) has gained the attention of the industry and research domain [1]. A number of research projects headed by different institutes and industry have been focusing on IoT application and service provision for health monitoring [2].

In the domain of smart homes, numerous sensor devices are used to support Ambient Assisted Living (AAL) such as motion and fall detectors, smoke alarms, bed detectors and others [3]. These devices are usually used to monitoring events related to the user's healthcare condition or falls and notifying a caregiver or a member of the family about an emergency incident. Thus, the receiver of the alert service is responsible to trigger the emergency call and provide information regarding the emergency event such as the symptoms, the cause, location, and the severity of the incident, information that most of the times does not even know. Moreover, the intervention of the intermediate receiver increases the response time that may lead to increased costs due to hospitalization or unpredictable loses of human life. In emergency cases, the time between the occurrence of the event and the service provision is crucial and should be eliminated. Enabling IoT and sensor devices to generate automatically emergency calls is a challenging issue for responding in an efficient thus accurate way to emergency incidents and avoiding unreasonable waste of time.

In this paper, an emergency framework (IoTA framework) is proposed that meets all the aforementioned challenges by generating automatically emergency calls to the appropriate healthcare provider and/or public safety answering point (PSAP). IoTA framework incorporates sensors that monitor the vital-signs and the body position of people suffering from diverse chronic diseases such as seizures in order to detect abnormal events. It incorporates outlier detection algorithms for filtering the aggregated data and identifying if the abnormal event occurs due to an emergency event. Whenever an alarm is triggered due to an abnormal event, it uses intelligent decision algorithms in order to distinguish its type and severity. Then, it forwards the sensor data to a SIP client (i.e. SIP phone) that establishes an emergency call with the appropriate healthcare provider supplying information about the location of the event, sensor data, video and voice. Using decision algorithms based on the set of the available sensor devices eliminates the hoax calls triggered by a single sensor. Moreover, it supports mechanisms that enable the call taker of the emergency call to request continuous sensor data using a bi-directional communication with the IoT devices.

A prototype application has been implemented as a proof of concept in order to demonstrate the basic features and the effectiveness of the proposed system. The case study used for evaluation purposes is based on a real-time scenario for monitoring patients suffering from seizures and need help immediately in order to avoid injuries. However, the proposed solution can be easily applicable to many IoTbased solutions such as ecall systems or smart cities in order 
to perform emergency calls.

The rest of the paper is organized as follows: in section II is presented the related work. The proposed IoTA framework architecture is presented in section III. In Section IV and V, the implementation details and a qualitative evaluation of the proposed IoTA framework are presented and discussed respectively. Finally, Section VI concludes the whole paper.

\section{RELATED WORK}

The World Health Organisation (WHO) [4] refers that seizure episodes are a result of excessive electrical discharges in a group of brain cells. Seizures can vary in frequency and in the part of the brain the disturbance first starts. Temporary symptoms occur, such as loss of awareness or consciousness, and disturbances of movement, sensation, mood, or other cognitive functions. One seizure does not signify epilepsy, a number of conditions can imitate seizures such as mini-strokes, hypoglycemia, panic attacks, etc. People with seizures tend to have more physical problems (such as fractures and bruising from injuries related to seizures)[4]. Studies report that providing emergency services in an accurate way is crucial and can reduce the impact of accidents. For a noticeable reduction of the response time, is mandatory the fast and accurate detection of the emergency event and the reporting to the appropriate healthcare provider in order to receive immediately emergency services.

In the healthcare domain, IoT devices have been used in order to detect or prevent seizures. Many algorithms and intelligent algorithms have been proposed that evaluate the electroencephalography (EEC) data received from the user's scalp, electromyography (EMG) or electrocardiogram (ECG) data. Cook et al. [5] proposed an intracranial implanted device used to collect EEG data on the cortical surface. The electrode leads were connected to an implanted telemetry unit, which wirelessly transmitted data to an external, handheld personal advisory device. The external device received the EEG data, applied an algorithm to them, and displayed the resultant information to the user's device. Authors in [6], [7] and [8] have proposed the use of accelerometers either wrist-worn or limb and chest worn for detecting generalized tonicclonic seizures. Different mechanisms have been implemented using linear thresholds, standard deviations, short-time Fourier transform, pattern recognition algorithms or time-domain and frequency-domain based algorithms in order to identify the existence of a seizure event [9]. In all these works, once a seizure was detected by the algorithm, the control unit triggered an alarm calling for help. The alarm was sent to the caretakers.

Seizures can be unpredictable. Some may start with minor symptoms, and then lead to a fall or a loss of consciousness. In this context, fall detection algorithms will be useful in order to detect the health status of the person experience a seizure. In the bibliography, there are various fall detection systems and classification algorithms have been proposed and evaluated in order to increase the accuracy of the detection or prevent a fall. Yazar, Erden and Cetin [10] proposed a low-cost and privacy-friendly fall detection system for AAL using vibration and passive infrared (PIR) sensors. Yanuz et al [11] proposed an enhanced smartphone-based fall detection system with location information using Google Maps about the person experienced the fall. When a fall is detected, the available GPS interface on the smartphone transmits the notification of the fall incident to the caregiver via SMS, email and Twitter messages. Habib et al [12] proposed a smartphone-based fall detection and prevention system for fall management. Combinations of alarm systems and graphical user interface are used for collecting the feedback of the user. If the user does not respond within that time, the system will consider the event as a fall and generate several types of notifications to seek help from caregivers (e.g. SMS, MMS, email,twitter messaging, etc). Sposaro and Tyson [13] proposed a similar system for fall detection using common commercially available electronic devices to both detect the fall and alert authorities.If a fall is suspected a notification is raised requiring the users response or a confirmation of user's social contact about the event.

The aforementioned works have been focused on detecting either seizures or falls using a series of sensor devices wearable or incorporated into smartphones and notifying user's caregivers and relatives. The caregivers are responsible for triggering the emergency call systems and reporting information regarding the emergency event. The existence of reporting to the caregivers an emergency call increases the response time that may lead to unpredictable loses of human life. To the best of our knowledge, the work of Cirani, Picone and Veltri [14], was the first that enabled IoT devices to perform SIP-based communication sessions in a lightweight and standard fashion. Then, NEXES [15] project proposed the integration of the sensor devices using the MSD format in order to establish automatically SIPbased emergency calls.

In this paper, we propose IoTA framework that meets all the aforementioned challenges by generating automatically emergency calls to the appropriate healthcare provider. IoTA framework is not another framework that promises to prevent seizures but it is totally differentiated from the above mentioned works in three basic ways. Firstly, it correlates data from multiple sensors that identify the existence of an emergency event due to seizure or fall and it can support the user during and after the emergency event triggering automatically healthcare providers. Moreover, it performs communication sessions directly with the healthcare providers forwarding the sensor data to a SIPbased client that establishes an emergency call with them. Healthcare providers can have access and request continuous data transmission in order to assist the patient in an accurate way. Finally, contrary to the CoSIP work [14], IoTA is independent of the application protocol that sensors use to 
transmit data.

\section{IOTA FRAMEWORK ARCHITECTURE}

The IoTA Framework is located at the caller's side. It comprises the sensor platform, the sensor data interface and the SIP client as presented in Fig.1. The caller's side can be also equipped with various appliances (i.e. laptops, tablets, TV sets, microphones, speakers), environmental sensors (i.e. humidity, smoke detector, motion) and healthcare monitoring sensors (i.e. pulsioximeter, thermometer, ECG). These sensors monitor the environmental conditions and bio-signals that indicate the health status of the caller.

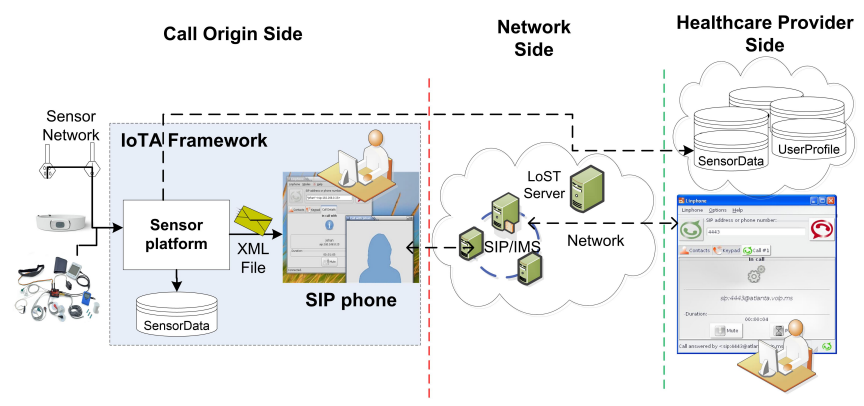

Figure 1. The IoTA Framework architecture overview.

The sensor platform is responsible for aggregating environmental, medical data and the user's position from multiple sensors. These data are stored either to an internal SensorDatabase for processing or to an external cloud base on the healthcare provider's side. Sensor platform incorporates multiple algorithms for processing and evaluating the sensor data so as to detect abnormal events and identify their type, if it is an outlier value or an emergency event. As an emergency event is detected, the sensor platform creates an alert and sends the sensor data in an XML-format to the SIP client i.e. SIP-based VoIP application - SIP phone) establishing a communication session with the appropriate healthcare provider.

The SIP Client is a key component for emergency services as it supports location information and routing to the appropriate healthcare provider. It is enhanced in order to use Uniform Resource Names (URNs) identifiers for IP-based emergency calls and support SIP SUBSCRIBE/NOTIFY messages. The URN service allows the identification of an emergency service to be triggered for each type of emergency event (i.e. ambulance, emergency room, etc) overlooking the need to manually search for the provider of that service. The implementation of the URN in the SIP client enables accurate report to the appropriate provider eliminating time delays due to manual search for the provider of that service. Moreover, in the IoTA Framework the SIP client is envisioned to have an additional interface to sensor platforms so as to provide enhanced functionalities by reporting the status of the emergency caller. In this context, the SIP client supports the SIP SUBSCRIBE/NOTIFY message [16] providing a bi-directional communication between the citizens, their IoT devices and the healthcare providers. This di-directional communication enables the call taker to be informed in an accurate way and request continuous realtime sensor data streaming in order to detect the emergency event, estimate its severity and respond swiftly and effectively, saving lives.

The sensor data interface has been developed as a client server model in order to enable the communication between the sensor platform and the SIP client. The interface accepts the critical data in XML format. Sensor Model Language (SensorML) [17] has been selected for standard based, unified sensor data representation model. Then, it encapsulates them in the body of SIP messages and forwards them to the healthcare provider through normal SIP routing. This approach is in conformity with already standardized solutions, like PIDF-LO (RFC 513943), and thus can be efficiently adapted to existing solutions.

\section{A. Sensor Platform}

The sensor platform is composed of the following components (Fig. 2):

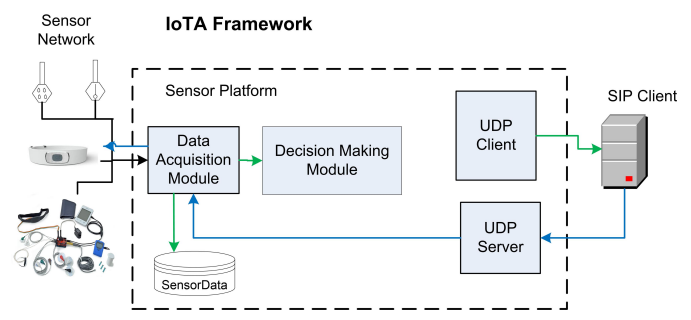

Figure 2. The components of the sensor platform architecture.

- Data Acquisition Module: it provides two interfaces for receiving data. The former enables real time sensor data aggregation and storing and the latter one allows data retrieval for updating purposes.

- Decision Making Module: it processes and evaluates real time data received from medical sensors in order to detect emergency events. As soon as an abnormal event is detected, it implements algorithms to detect either possible sensor failures or actual emergency incidents, creates an alarm and forwards the sensor data regarding the emergency event to the UDP Client component.

- UDP Client: This component creates an XML file that contains the sensor data received from the Decision Making Module or Data Acquisition Module component and sends it over UDP/IP protocol to a SIP client.

- UDP Server: This component provides an interface to the SIP client that allows the PSAP responders to request updated real time data of the caller that initiated the emergency call. The UDP Server receives SIP messages. 


\section{B. SIP Client}

The SIP client consists of the following components (Fig.3):

- UrnMapping: This sub-component performs the translation of emergency number, such as 112 , to the corresponding URN service identifier (e.g., urn:service:sos).

- SensorCommunication: This component implements the communication interface to the sensor platform. It is based on the MESSAGE SIP method [18] and exploits UDP as transport. It receives an alert in the case of an abnormal medical event and triggers the InitiateEmergencyCall. Whenever an update of the sensor values is required, the component is also able to make a request to the sensor platform and forward the collected data to the SensorUpdateEvent component.

- InitiateEmergencyCall: It initiates an enhanced emergency call. In this case, the medical data are included in the body of the INVITE message in SensorML format [17]. It also includes the provided URN identifier in the To: field of the INVITE message.

- SensorUpdateEvent: This component supports the update of the sensor values during an emergency event at the client-side. For that purpose the SIP SUBSCRIBENOTIFY framework [16] is utilized, in order to allow the involved parts to get near real-time updated values of the callers sensor data (i.e. vital signs and environmental data). The SIP specific event notification framework allows the entities of the network (e.g., PSAP, first responders) to subscribe to the state of other entities, which in return can send notifications when their state changes.

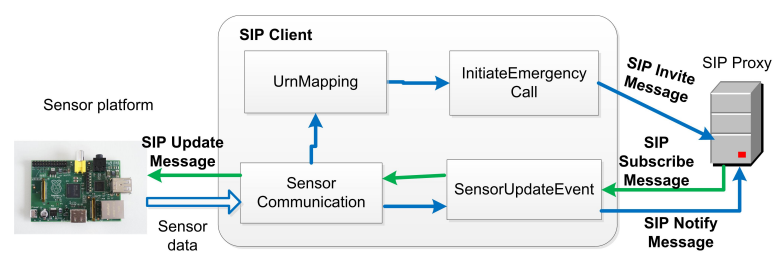

Figure 3. The components of the SIP Client.

\section{IMPLEMENTATION}

\section{A. System SetUp}

A prototype application was developed as a proof of concept for IoTA Framework. The application includes two main components: the sensor platform and the SIP Client.

The sensor platform is developed upon a low power single-board computing device (Raspberry Pi2) running Debian operating system and using Ethernet or IEEE 802.11 wireless connection to the Internet. The software of the sensor platform is developed in C++ multithreading programming model. The software that collects data from each sensor is implemented and runs as an independent thread enabling fast execution of the outlier detection and decision processes and optimizing resource consumption, latency and responsiveness. The sensor data are stored locally to a database located at Pi for temporary usage and to a cloud database located to the healthcare provider for permanent storage. The InfluxDB database [19] was used in both cases. An open source metric analytics and visualization tool, Grafana [20] was used in both user and healthcare provider's end in order to visualize time series data from sensors.

The SIP Client is implemented based on the opensource Voice Over IP phone, Linphone [21]. SIP Client was extended in order to encapsulate information regarding the location and the sensor data. SIP Client retrieves the user's location from location servers. The LoST (Location to Service Translation) protocol [22] which maps URNs and location information to one or more service URLs is used to retrieve the location of the appropriate healthcare provider. SIP Client retrieves the location by the providers using a PIDF-LO XML payload and encapsulates it into the SIP INVITE message for initiating an emergency call. Moreover, similar to the location data, SIP Client receives the aggregated bio-signals from sensors into an XML-format which is encapsulated to the SIP INVITE message body. SIP Client developed in C, runs on Ubuntu 14.04 operating system.

\section{B. Decision Process}

The decision process is divided into two discrete phases (Fig. 4). In the first phase, the readings of the sensors are evaluated in order to prune the outlier values. In the second phase of the decision process, a hybrid emergency detection algorithm is proposed that detects falls and correlates biosigns data in order to detect emergency events. Whenever an emergency event is identified, sensor data are sent to the SIP Client triggering an emergency call.

1) Outlier Detection: IoT and sensory devices send data and vital-signs to the sensor platform. The software of each sensor acquires and processes the incoming data of the sensor at a rate of 1 measurement per second. For each sensor, predefined threshold values are used in order to detect abnormal events. As normal values we define values that their distance from the other time-series data is greater than the threshold margin. When an abnormal event is detected, a time-series outlier detection algorithm is performed in order to discern conditions between a faulty sensor reading and a patient entering into an emergency state. The outlier detection algorithm uses the median value of an x-time-series data to determine whether the current sensor reading is an outlier (Algorithm 1).

2) Hybrid Emergency Detection Algorithm: If the abnormal value is not labeled as outlier, a three-phase hybrid emergency detection algorithm is performed distinguishing the type (fall, seizure or both) and the severity of the emergency. The one-phase algorithm evaluates data from 


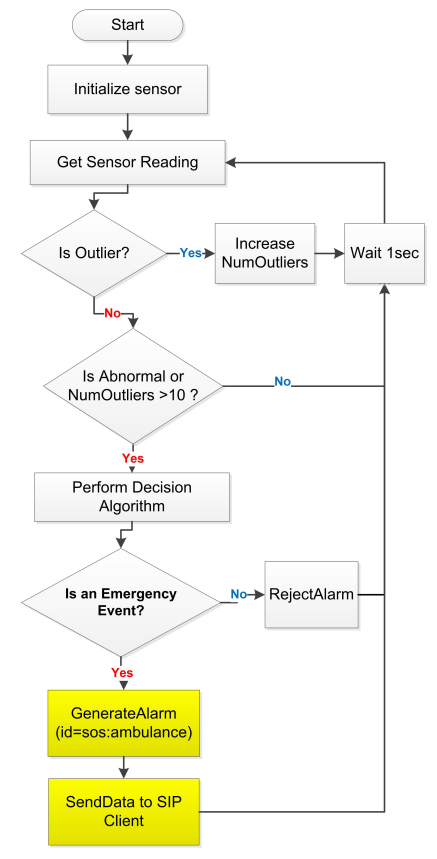

Figure 4. The flow diagram of the decision process.

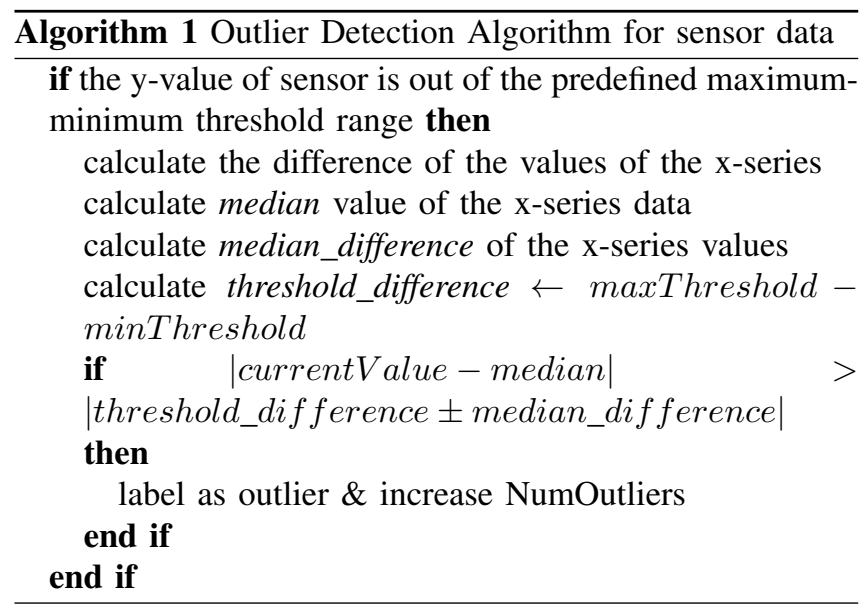

accelerometer and gyroscope for detecting falls. We used the algorithm proposed by [23] due to the high accuracy $(99.382 \%)$ in a timely manner that performs in contrary to other more complex algorithms. In the second-phase, motivated by the results presented in [24], we correlated bio-signals from the medical sensors (ECG, blood pressure, pulsioximeter, body thermometer, airflow) using J48 classification algorithm in order to detect seizure and/or stroke [25]. The J48 classification algorithm generates decision trees, the nodes of which evaluate the existence or significance of a seizure or stroke event. A decision-tree model is built by analyzing training data and the model is used to classify unseen data. In our model, the tree nodes are the monitored sensor data and the leaf nodes are the class (seizure, stroke, normal). Then, using a rule-based algorithm we decide for the generation of the emergency alarm (Algorithm 2).

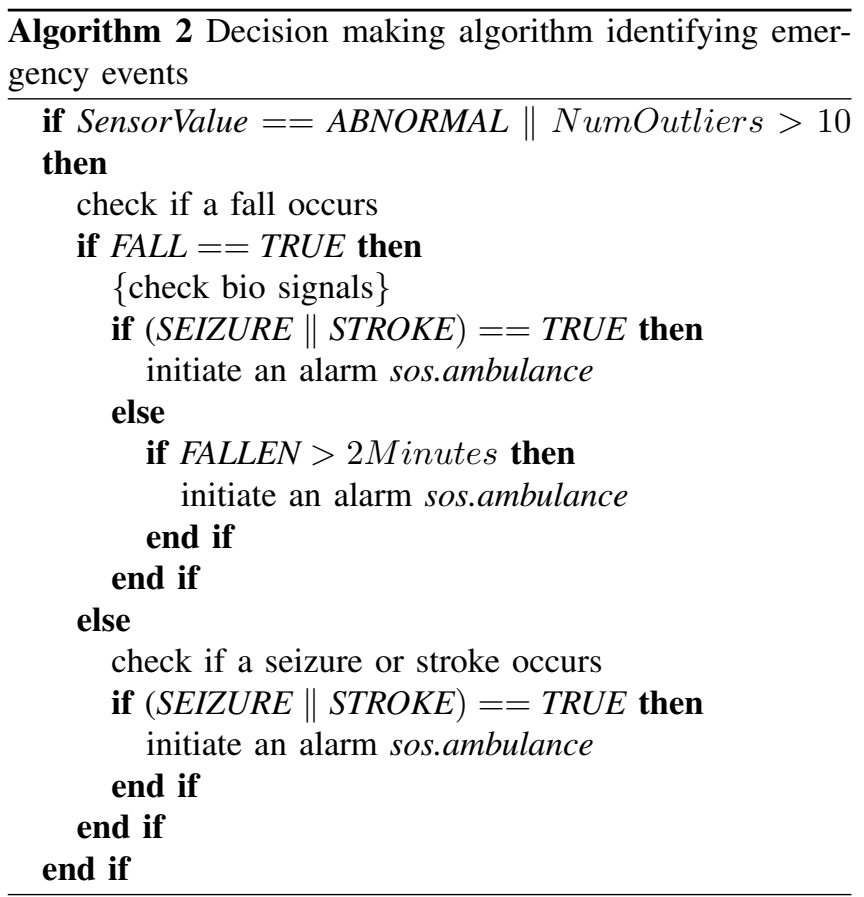

\section{Communication Establishment}

The sensor platform aggregates, processes and evaluates the sensor data. Whenever an emergency incident is detected, the sensor platform device sends the sensor data related to the emergency event using the SensorML [17] format to the SIP client via the UDP Client. The combined sensor data are sent as part of the SIP INVITE message via the Linphone application running on user's laptop or smartphone. The recipient of the emergency call is able to request continuous updating of the sensor data. To support this, the sensor platform incorporates a UDP Server. The UDP Server running on the sensor platform receives messages from the SIPbased application, parses the header, stores the destination and source IP addresses as well as the CallID and the time interval dictated by the healthcare provider to receive updated sensor data. Then, it forwards to the SIP client the updated sensors data until it receives the message to stop forwarding. Fig. 5 presents the communication establishment between the sensor platform and the SIP Client.

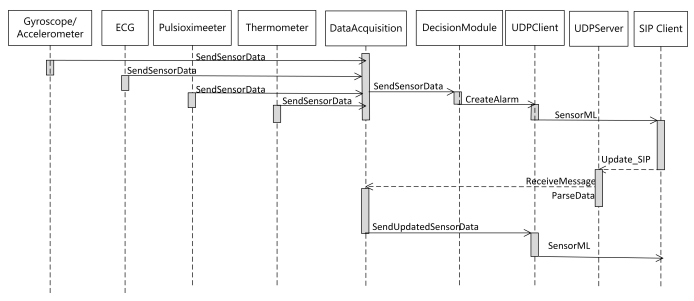

Figure 5. The sequence diagram of the internal communication establishment of IoTA Framework. 
Through the SIP client, each emergency event enters the system and joins the appropriate healthcare according to its type of alarm and location. The SIP client is responsible for establishing and maintaining the SIP communication session and updating the sensor data to the healthcare side through SIP SUBSCRIBER/NOTIFY method. Whenever SIP Client is triggered by the sensor platform, SIP CLient retrieves the user's location from location service providers (DHCP, HELD, LLDP-MED, Android Location Services). SIP Client receives the location information in a PIDF-LO XML format and encapsulates it in a SIP INVITE. SIP INVITE message encapsulates to its payload also data from the sensors in an XML format. SIP INVITE message and is sent to the SIP Proxy. SIP proxy is extended to support emergency services recognition and routing. It receives a SIP call with a URN indicating the emergency service requested (i.e. ambulance) requests from a location service to determine the appropriate healthcare provider to route the call. Then the call is forwarded to the SIP Client of the healthcare provider (the recipient of the call). For the event notification, the SIP specific event notification framework is used so as to allow emergency stakeholders (e.g. healthcare providers, first responders) to subscribe to the state of other entities, which in return can send notifications when their state changes. Fig. 6 presents the communication establishment between the SIP Client and the healthcare provider.

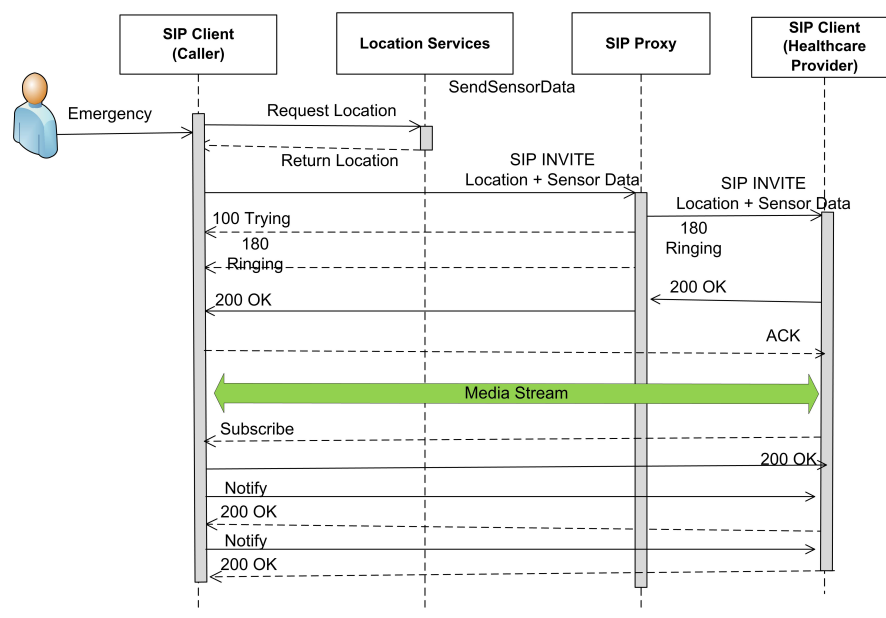

Figure 6. The sequence diagram of the inter communication establishment of IoTA Framework.

\section{System Demonstration}

In Fig. 7 is presented the screenshot during the automatically emergency triggering process. The IoTA framework initiated an automated triggering call due to a fall that lasted more than 2 minutes. A multimedia communication session is established with the healthcare and the sensor data as well as the location information are presented in a user-friendly Graphical User Interface (GUI).

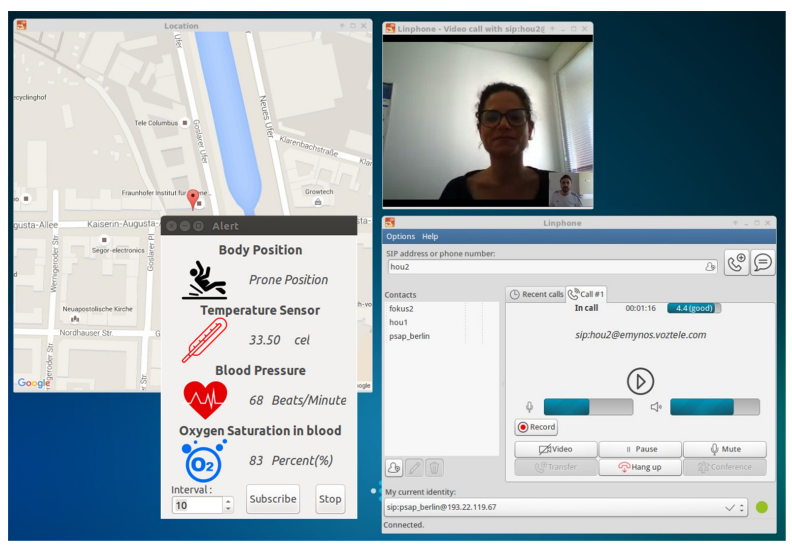

Figure 7. Screenshot of IoTA framework triggering an emergency call to the healthcare Provider.

\section{EVALUATION}

In order to support patients suffering from diverse chronic diseases that may experience a seizure (i.e. epilepsy, ischemic stroke) a set of lightweight sensors are provided to the user. These sensors include body thermometer, pulsioxemeter, ECG, blood pressure, airflow and galvanic Skin response sensors. A tri-axial accelerometer ADXL345 and a gyroscope ITG3200 were used in order to measure acceleration and angular velocity for detecting falls. The accelerometer/gyroscope is packed into a chest belt for optimum location retrieval. All the sensors are connected directly to the sensor platform. The sensor platform is a wireless portable device carried into the user's pocket.

The experiment is performed on data from 15 elderly healthy and non-healthy subjects (6 men and 9 women, aged from 60 to 75 years). The data emulate subjects that monitor their healthcare status using the medical sensors and simultaneously perform their daily activities including walking, ascending/descending stairs, sitting, standing or sleeping.

In order to evaluate the effectiveness of the proposed system, a logging/auditing service was implemented on the sensor platform. It is activated when a sensor value is received and creates log files reporting the number of the abnormal values before and after the performance of the outlier detection and the decision algorithms are counted so as to identify the accuracy of of IoTA framework. The accuracy and the error hereafter hoax calls are defined as:

where:

$$
\begin{gathered}
\text { Accuracy }=\frac{T P \text { Alarm }}{T P \text { Alarm }+F N A l a r m} \\
\text { HoaxCalls }=\frac{T N \text { Alarm }}{T N \text { Alarm }+F P \text { Alarm }}
\end{gathered}
$$

- TPAlarm: it is the number of true positive alarms by means of emergency event occurred and detected by IoTA.

- FPAlarm: it is the number of false positive alarms meaning that IoTA detects an emergency event which does not occur. 
- TPAlarm: it is the number of true negative alarms meaning that there is not any emergency event and IoTA detects it as normal (non-emergency)event.

- FNAlarm: it is the number of false negative alarms meaning that an emergency event occurs and IoTA does not detect it.

By observing the results shown in Table I, it comes out that incorporating multiple sensors and decision schemes has reduced the number of hoax calls. This is due to the fact that inherently, the data retrieved by a single sensor are not reliable to identify emergency events. It should be mentioned that the accuracy of the fall, seizure or stroke detection algorithm (presented in Table I) is related to their effectiveness to identify an emergency event. This is totally different from their effectiveness to identify the event for which they are implemented. For instance, during a seizure the person loses his consciousness and falls. In this case, the fall and seizure detection algorithm would trigger an alarm. However, if the seizure lasts less than 5 minutes and the fallen person is not injured, there is no need to trigger an emergency call. The proposed decision algorithm takes into consideration these parameters leading to a more robust and effective emergency detection system (accuracy $82.86 \%$ ) eliminating the hoax calls which are estimated to $17.14 \%$.

Table I

THE ACCURACY OF IOTA FRAMEWORK TO DETECT ABNORMAL EVENTS AND TRIGGER A CALL.

\begin{tabular}{l|l|l}
\hline Abnormal events identified & Accuracy \% & $\begin{array}{l}\text { Hoax } \\
\text { Calls(\%) }\end{array}$ \\
\hline Using the outlier algorithm (Algorithm 1) & 20.68 & 79.32 \\
\hline Using fall detection algorithm & 45.21 & 64.79 \\
\hline $\begin{array}{l}\text { Using seizure and stroke detection algo- } \\
\text { rithms }\end{array}$ & 63.83 & 36.17 \\
\hline $\begin{array}{l}\text { Using the proposed decision algorithm (Al- } \\
\text { gorithms 1 \& 2) }\end{array}$ & 82.86 & 17.14 \\
\hline
\end{tabular}

Moreover, the auditing service creates log files reporting the processing time the oultier and decision algorithm require in order to identify an emergency event. Table II presents the average times in order to evaluate the received sensor data, perform decision algorithms and send the data to a pre-defined format ([17]) to the SIP client. The average time to identify if an abnormal value is an outlier, is estimated between 1 and $2 \mathrm{sec}$. This is due to the fact that the outlier detection algorithm verifies that an outlier exists after performing the same process for the next incoming value. Each sensor is implemented to send data to the sensor platform under a predefined time that varies from 1 to 2 seconds. As a consequence, when the second value arrives at the sensor platform, it is processed. The hybrid emergency detection algorithm performs an internal evaluation and verification process estimating the existence of an emergency case. The average total time is estimated at about 11.5 secs.
Table II

EVALUATION RESULTS OF SENSOR PLATFORM

\begin{tabular}{l|l}
\hline Process Evaluation & $\begin{array}{l}\text { Average } \\
\text { Time (s) }\end{array}$ \\
\hline $\begin{array}{l}\text { Outlier Algorithm processing } \\
\text { Hybrid emergency detection Algorithm pro- } \\
\text { cessing }\end{array}$ & 1.5 \\
\hline Total Response & 11.5 \\
\hline
\end{tabular}

For the initiation of a SIP session, the signaling cost for the extended SIP INVITE message encapsulated with data from six different sensors is equal to 1128 bytes while the SIP NOTIFY is equal to 582 bytes. As the number of sensors is increased, the length of the SIP INVITE and NOTIFY message is increased. Considering the CoSIP approach [14] that enables each sensor to generate an alarm, for a small number of sensors, only two, the extended SIP INVITE message overhead (670 bytes) is similar to this provided by CoSIP (2sensors $* 311$ CoSIPbytes/sensor $=622$ bytes $)$ [14]. However, for multiple sensors the extended SIP INVITE yields the CoSIP with a compression ratio above 0.5 (Table III). The signaling cost of the SIP communication and the average time required to establish a communication session with the healthcare provider is planned to be evaluated in future extensions of this work.

Table III

COMPARISON BETWEEN EXTENDED SIP AND COSIP MESSAGE OVERHEAD FOR SIP INVITE MESSAGE.

\begin{tabular}{l|l|l|l}
\hline $\begin{array}{l}\text { Number of } \\
\text { Sensors }\end{array}$ & $\begin{array}{l}\text { extended SIP } \\
\text { (bytes) }\end{array}$ & CoSIP (bytes) & $\begin{array}{l}\text { compression } \\
\text { ratio }\end{array}$ \\
\hline 2 & 670 & 622 & 1.077 \\
4 & 937 & 1244 & 0.753 \\
6 & 1128 & 1866 & 0.605 \\
\hline
\end{tabular}

\section{CONCLUSION}

In this paper, IoTA framework is proposed that enables IoT devices to generate automatically emergency calls to healthcare providers supporting people suffering from chronic diseases that live independently. IoTA framework aggregates and evaluates sensor data in order to detect abnormal events. It incorporates outlier detection algorithms and decision making algorithms that identify if the abnormal event is an emergency event or not. Whenever an alarm is triggered due to an emergency event, the IoTA framework forwards the sensor data to a SIP client that is responsible for establishing emergency calls with the appropriate healthcare provider providing a set of location and sensor data information related to the incident. The SIP client is enhanced in order to use the URN service identifier for IP-based emergency calls and support SIP SUBSCRIBE/NOTIFY messages. In this context, the call taker can request continuous real-time sensor data using a bi-directional communication with the IoT devices.

A prototype application was implemented as a proof of concept in order to demonstrate the basic features and the 
effectiveness of the proposed system supporting patients during and after a seizure. The evaluation results illustrate that the IoTA Framework can be considered as a promising solution for detecting, reporting emergency events and responding swiftly saving lives. The proposed solution can be easily incorporated to many IoT-based solutions so as to perform emergency calls and respond effectively in emergency incidents such as ecall systems or smart cities.

\section{ACKNOWLEDGMENT}

This work is supported by H2020 European Framework Programme under the EMYNOS - nExt generation eMergencY commuNicatiOnS project.

\section{REFERENCES}

[1] L. Atzori, A. Iera, and G. Morabito, "The internet of things: A survey," Computer networks, vol. 54, no. 15, pp. 2787-2805, 2010.

[2] N. Bui and M. Zorzi, "Health care applications: a solution based on the internet of things," in Proceedings of the 4th International Symposium on Applied Sciences in Biomedical and Communication Technologies. ACM, 2011, p. 131.

[3] A. Pantelopoulos and N. G. Bourbakis, "A survey on wearable sensor-based systems for health monitoring and prognosis," IEEE Transactions on Systems, Man, and Cybernetics, Part C (Applications and Reviews), vol. 40, no. 1, pp. 1-12, 2010.

[4] (02/2017) Epilepsy. [Online]. Available: http://www.who.int/ mediacentre/factsheets/fs999/en/

[5] M. J. Cook, T. J. O’Brien, S. F. Berkovic, M. Murphy, A. Morokoff, G. Fabinyi, W. D'Souza, R. Yerra, J. Archer, L. Litewka et al., "Prediction of seizure likelihood with a long-term, implanted seizure advisory system in patients with drug-resistant epilepsy: a first-in-man study," The Lancet Neurology, vol. 12, no. 6, pp. 563-571, 2013.

[6] J. Lockman, R. S. Fisher, and D. M. Olson, "Detection of seizure-like movements using a wrist accelerometer," Epilepsy \& Behavior, vol. 20, no. 4, pp. 638-641, 2011.

[7] U. Kramer, S. Kipervasser, A. Shlitner, and R. Kuzniecky, "A novel portable seizure detection alarm system: preliminary results," Journal of Clinical Neurophysiology, vol. 28, no. 1, pp. 36-38, 2011.

[8] S. Beniczky, T. Polster, T. W. Kjaer, and H. Hjalgrim, "Detection of generalized tonic-clonic seizures by a wireless wrist accelerometer: a prospective, multicenter study," Epilepsia, vol. 54, no. 4, 2013.

[9] S. Ramgopal, S. Thome-Souza, M. Jackson, N. E. Kadish, I. S. Fernández, J. Klehm, W. Bosl, C. Reinsberger, S. Schachter, and T. Loddenkemper, "Seizure detection, seizure prediction, and closed-loop warning systems in epilepsy," Epilepsy \& behavior, vol. 37, pp. 291-307, 2014.

[10] A. Yazar, F. Erden, and A. E. Cetin, "Multi-sensor ambient assisted living system for fall detection," in Proceedings of the IEEE International Conference on Acoustics, Speech, and Signal Processing (ICASSP14). Citeseer, 2014, pp. 1-3.
[11] G. Yavuz, M. Kocak, G. Ergun, H. O. Alemdar, H. Yalcin, O. D. Incel, and C. Ersoy, "A smartphone based fall detector with online location support," in International Workshop on Sensing for App Phones; Zurich, Switzerland, 2010, pp. 3135 .

[12] M. A. Habib, M. S. Mohktar, S. B. Kamaruzzaman, K. S. Lim, T. M. Pin, and F. Ibrahim, "Smartphone-based solutions for fall detection and prevention: challenges and open issues," Sensors, vol. 14, no. 4, pp. 7181-7208, 2014.

[13] F. Sposaro and G. Tyson, "ifall: an android application for fall monitoring and response," in Engineering in Medicine and Biology Society, 2009. EMBC 2009. Annual International Conference of the IEEE. IEEE, 2009, pp. 6119-6122.

[14] S. Cirani, M. Picone, and L. Veltri, "Cosip: a constrained session initiation protocol for the internet of things," in European Conference on Service-Oriented and Cloud Computing. Springer, 2013, pp. 13-24.

[15] Nexes: Next generation emergency services. [Online] Available: http://nexes.eu.

[16] A. Roach, "Sip-specific event notification," 2012.

[17] M. Botts, A. Robin, J. Greenwood, and D. Wesloh, "Ogc $\mathbb{R}$ sensorml: Model and xml encoding standard," Technical Standard, vol. 2, 2014.

[18] B. Campbell, J. Rosenberg, H. Schulzrinne, C. Huitema, and D. Gurle, "Session initiation protocol (sip) extension for instant messaging", rfc 3428," 2002.

[19] $(06 / 2017)$ Influxdb. [Online]. Available: https:// home-assistant.io/components/influxdb/

[20] (06/2017) Grafana labs: The analytics platform for all your metrics. [Online]. Available: https://grafana.com/grafana

[21] Linphone open source voip project. [Online]. Available: http://www.linphone.org/

[22] (2017) Emergency context resolution with internet technologies (ecrit). [Online]. Available: http://ecrit. sourceforge.net/lost_server.html

[23] Q. T. Huynh, U. D. Nguyen, S. V. Tran, A. Nabili, and B. Q. Tran, "Fall detection system using combination accelerometer and gyroscope," in Proc. of the Second Int. l Conf. on Advances in Electronic Devices and Circuits (EDC 2013), 2013.

[24] A. Van de Vel, K. Cuppens, B. Bonroy, M. Milosevic, K. Jansen, S. Van Huffel, B. Vanrumste, L. Lagae, and B. Ceulemans, "Non-eeg seizure-detection systems and potential sudep prevention: state of the art," Seizure, vol. 22, no. 5 , pp. $345-355,2013$.

[25] H. D. Masethe and M. A. Masethe, "Prediction of heart disease using classification algorithms," in Proceedings of the world Congress on Engineering and computer Science, vol. 2, 2014, p. 2224. 